\title{
铜催化亚磺酸钠盐参与的萗胺远程磺酰化反应
}

\author{
周翔†余茹鉴†王金涛廖向文熊艳师* \\ (江西科技师范大学药学院 江西省药物分子设计与评价重点实验室 南昌 330013)
}

\begin{abstract}
摘要 报道了一种简单且温和的铜催化荎-1-胺与亚磺酸钠和亚磺酸锂的磺酰化反应. 在反应以碘作为氧化剂, 在比较 温和的反应条件下，可以高选择性地实现菜环 C(4)的磺酰化反应，并且以中等至较高产率生成了各种砜类衍生物.

关键词 铜催化; 磺酰化; 远程 $\mathrm{C}-\mathrm{H}$ 官能团化
\end{abstract}

\section{Copper-Catalyzed Remote Sulfonylation of 1-Naphthylamides with Sodium-Sulfinates}

\author{
Zhou, Xiang $^{\dagger} \quad$ Yu, Rujian ${ }^{\dagger} \quad$ Wang, Jiantao Liao, Xiangwen $\quad$ Xiong, Yanshi* \\ (Jiangxi Provincial Key Laboratory of Drug Design and Evaluation, School of Pharmacy, Jiangxi Science \& Technology \\ Normal University, Nanchang 330013)
}

\begin{abstract}
A simple and mild copper-catalyzed sulfonylation of naphthalen-1-amine with sodium and lithium sulfinates is reported. In the presence of iodine as oxidant a highly site-selective $\mathrm{C}-\mathrm{H}$ functionalization at the $\mathrm{C}(4)$ position took place. The reaction proceeded readily mild conditions and various sulfones were synthesized in moderate to high yields.

Keywords copper-catalyzed; sulfonylation; remote $\mathrm{C}-\mathrm{H}$ functionalization
\end{abstract}

\section{Introduction}

Naphthalenes are important building blocks in organic synthesis and common structure motifs found in numerous pharmaceuticals and functional materials. ${ }^{[1]}$ For example, heteroaromatic sulfones are widely found in numerous potential drugs against various disease, such as human immunodeficiency virus (HIV) ${ }^{[2]}$ or neurological disorders. ${ }^{[3]}$ Efficient synthesis of sulfones compound has received tremendous interests. Traditional approaches for the synthesis of sulfones include the oxidation of sulfides, ${ }^{[4]}$ the alkylation of sulfinates, ${ }^{[5]}$ Friedel-Crafts type sulfonylation of arenes with sulfonyl chlorides or addition reaction of sulfonyl-based radicals to olefins and alkynes. ${ }^{[6]}$ Although these strategies can sever as promising approaches for the synthesis of aryl sulfones, they all suffer from the need of using per-functionalized stating materials such as aryl halides. Apart from these methods, direct sulfonylation using the parent arenes as the starting materials may be attractive since no per-functionalization is required. During the past decades, transition-metal catalyzed or me- diated $\mathrm{C}-\mathrm{H}$ functionlization has become a facial tool in organic synthesis, ${ }^{[7]}$ and a series of transformations from $\mathrm{C}-\mathrm{H}$ to $\mathrm{C}-\mathrm{C}$ and $\mathrm{C}$-heteroatom bonds has been demonstrated. ${ }^{[8]}$ However, comparing with the widely studied directing group-assisted $\mathrm{C}-\mathrm{H}$ functionalization of substituted benzenes, reports on $\mathrm{C}-\mathrm{H}$ functionalization on the naphthalene ring systems are relatively rare. ${ }^{[9]}$ Several examples were focused on the transition metal-catalyzed/ mediated $\mathrm{C}-\mathrm{C},{ }^{[10]} \mathrm{C}-\mathrm{O}^{[11]}$ and $\mathrm{C}-\mathrm{N}^{[12]}$ bonds formation at the neighboring ortho- and peri-positions of the directing groups. The more challenging $\mathrm{C}-\mathrm{S}$ bond formation is largely underexplored, mainly because of the strong coordination of the metal catalysts to the sulfur reagents. Our group ${ }^{[13]}$ and Nishihara's group ${ }^{[14]}$ have reported copper-catalyzed and palladium-catalyzed peri-selective direct sulfonylation of 1-naphthylamine with diphenyl disulfide, however they didn't get para-selective products. In our continuous research on direct $\mathrm{C}-\mathrm{S}$ bond formation by base metal catalysts, we focus on developing methods for the sulfonylation of 1-naphthylamines $\mathrm{C}(4)-\mathrm{H}$ bonds in consideration of the great challenges associated. In recent,

\footnotetext{
* Corresponding author. E-mail: xiongys1214@163.com

Received June 15, 2021; revised July 21, 2021; published online August 17, 2021.

Project supported by the Department Education Science and Technology Research Project of Jiangxi Province (Nos. GJJ190619, GJJ201143), and the PhD Research Startup Foundation of Jiangxi Science \& Technology Normal University (No. 2019BSQD016).

江西省教育厅青年项目(Nos. GJJ190619, GJJ201143)和江西科技师范大学博士科研启动基金(No. 2019BSQD016)资助项目.

†共同第一作者(These authors contributed equally to this work).
} 
Manolikake's group $^{[15]}$ and Wu's group ${ }^{[16]}$ have independently reported the remote para-selective sulfonylation of 1-naphthylamines catalyzed by $\mathrm{Cu}(\mathrm{OAc})_{2}$ through bidentate-chelation induced single-electron-transfer (SET) pathway. However, those methods not only needed amount of metal oxidant, but also required sliver cocatalyst and other additives. Herein we disclose the aerobic coppercatalyzed remote sulfonylation of 1-naphthylamines to prepare 4-sulfenyl-1-naphthylamines, which are important structural motifs present in bioactive molecules with anticancer and antibacterial properties. ${ }^{[17]}$

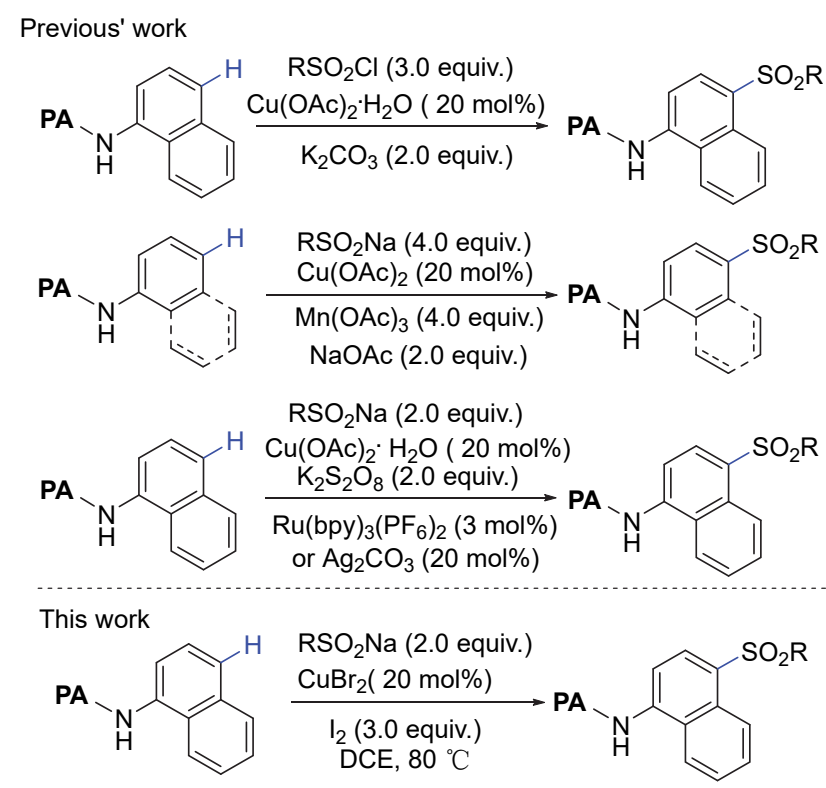

Scheme 1 Direct C(4)-SO $\mathrm{SO}_{2}$ bond formation of 1-naphthylamides

\section{Results and discussion}

To explore the possibility of our expected transformation, we commenced our study with $N$-(naphthalen-1yl)-picolinamide (1a) and sodium benzenesulfinate (2a) as the model substrates (Table 1). To our delight, the desired $\mathrm{C}(4)$ sulfonylated product $3 \mathbf{a}$ was obtained in $20 \%$ yield in the presence of $\mathrm{CuBr}_{2}(20 \mathrm{~mol} \%)$ with $\mathbf{2 a}$ (2.0 equiv.) in 1,2-dichloroethane (DCE) $2 \mathrm{~mL}$ under air at $80{ }^{\circ} \mathrm{C}$ for 12 h (Table 1, Entry 1). The molecular structure of 3a was confirmed by NMR spectra. The yield of $\mathbf{3 a}$ could be increased up to $55 \%$ by employing $\mathrm{I}_{2}$ (2.0 equiv.) as the oxidant (Table 1, Entry 2), and then several oxidants were examined, as shown in Table 1 (Entries 3 9), the sulfonylated product 3a was obtained with isolated yield up to $89 \%$ when $\mathrm{I}_{2}$ (3.0 equiv.) was employed. Motivated by this result, we screened a series of copper salts and found that changing the oxidation state or the counterion of the salt could also execute this transformation, albeit with lower efficiencies (Table 1, Entries 10 14). Further screening showed that DCE is the most suitable solvent for the reaction (Table 1, Entries 16 21). However, the desired product 3a was only obtained in $66 \%$ yield when we lowering the reaction temperature from 80 to $60{ }^{\circ} \mathrm{C}$, and the reaction time as longer to $16 \mathrm{~h}$ was needed (Table 1, Entry 22). In addition, the control experiment showed that no product was formed when the reaction was performed in the absence of the copper salt (Table 1, Entry 15), or the yield was reduced to $75 \%$ in the present of $10 \%$ of $\mathrm{CuBr}_{2}$ (Table 1, Entry 23). Consequently, we decided to set reacting 1a with $20 \mathrm{~mol} \%$ of $\mathrm{CuBr}_{2}$, sodium sulfinates $(2.0$ equiv.) and $\mathrm{I}_{2}$ (3.0 equiv.) in DCE at $80{ }^{\circ} \mathrm{C}$ for $12 \mathrm{~h}$ as the standard conditions (Table 1, Entry 9).

Table 1 Optimization of the reaction conditions ${ }^{a}$

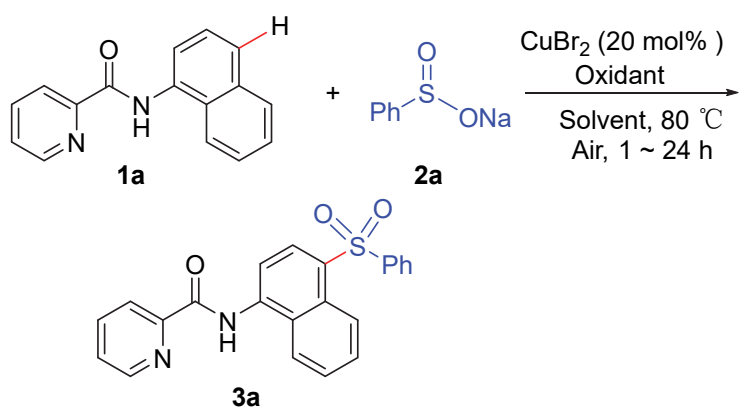

\begin{tabular}{|c|c|c|c|c|}
\hline Entry & Catalyst & Oxidant (equiv.) & Solvent & Yield ${ }^{b} / \%$ \\
\hline 1 & $\mathrm{CuBr}_{2}$ & - & DCE & 20 \\
\hline 2 & $\mathrm{CuBr}_{2}$ & $\mathrm{I}_{2}(2.0)$ & DCE & 55 \\
\hline 3 & $\mathrm{CuBr}_{2}$ & TBHP (2.0) & DCE & 32 \\
\hline 4 & $\mathrm{CuBr}_{2}$ & DTBP (2.0) & DCE & 27 \\
\hline 5 & $\mathrm{CuBr}_{2}$ & $\begin{array}{l}\mathrm{PhI}(\mathrm{OAc})_{2} \\
(2.0)\end{array}$ & DCE & 40 \\
\hline 6 & $\mathrm{CuBr}_{2}$ & BQ (2.0) & DCE & 17 \\
\hline 7 & $\mathrm{CuBr}_{2}$ & $\mathrm{Ag}_{2} \mathrm{CO}_{3}(2.0)$ & DCE & 15 \\
\hline 8 & $\mathrm{CuBr}_{2}$ & $\mathrm{I}_{2}(1.0)$ & DCE & 37 \\
\hline 9 & $\mathrm{CuBr}_{2}$ & $\mathrm{I}_{2}(3.0)$ & DCE & 90 \\
\hline 10 & $\mathrm{Cu}(\mathrm{OAc})_{2}$ & $\mathrm{I}_{2}(3.0)$ & DCE & 35 \\
\hline 11 & $\mathrm{CuCl}_{2}$ & $\mathrm{I}_{2}(3.0)$ & DCE & 47 \\
\hline 12 & $\mathrm{Cu}(\mathrm{OTf})_{2}$ & $\mathrm{I}_{2}(3.0)$ & DCE & 27 \\
\hline 13 & $\mathrm{Cu}(\mathrm{OAc})_{2} \cdot \mathrm{H}_{2} \mathrm{O}$ & $\mathrm{I}_{2}(3.0)$ & DCE & 35 \\
\hline 14 & $\mathrm{CuO}$ & $\mathrm{I}_{2}(3.0)$ & DCE & Trace \\
\hline 15 & - & $\mathrm{I}_{2}(3.0)$ & DCE & Trace \\
\hline 16 & $\mathrm{CuBr}_{2}$ & $\mathrm{I}_{2}(3.0)$ & $\mathrm{CH}_{3} \mathrm{CN}$ & 57 \\
\hline 17 & $\mathrm{CuBr}_{2}$ & $\mathrm{I}_{2}(3.0)$ & THF & 33 \\
\hline 18 & $\mathrm{CuBr}_{2}$ & $\mathrm{I}_{2}(3.0)$ & DMSO & 67 \\
\hline 19 & $\mathrm{CuBr}_{2}$ & $\mathrm{I}_{2}(3.0)$ & DMF & 45 \\
\hline 20 & $\mathrm{CuBr}_{2}$ & $\mathrm{I}_{2}(3.0)$ & Toluene & 57 \\
\hline 21 & $\mathrm{CuBr}_{2}$ & $\mathrm{I}_{2}(3.0)$ & $\mathrm{MeOH}$ & 23 \\
\hline $22^{d}$ & $\mathrm{CuBr}_{2}$ & $\mathrm{I}_{2}(3.0)$ & DCE & 66 \\
\hline $23^{e}$ & $\mathrm{CuBr}_{2}$ & $\mathrm{I}_{2}(3.0)$ & DCE & 75 \\
\hline
\end{tabular}

${ }^{a}$ Reaction conditions: 1a $(0.2 \mathrm{mmol}), 2 \mathrm{a}(0.4 \mathrm{mmol}), \mathrm{Cu}(20 \mathrm{~mol} \%)$, oxidant (1.0 3.0 equiv.), solvent $(2 \mathrm{~mL})$, under air, $80{ }^{\circ} \mathrm{C}$ for $14 \mathrm{~h} ;{ }^{b}$ Yields of isolated product; ${ }^{c}$ Stirred at $60{ }^{\circ} \mathrm{C} ;{ }^{d}$ In the presence of $\mathrm{CuBr}_{2}(10 \mathrm{~mol} \%)$.

With the optimized reaction conditions in hand, the substrate scope with respect to sodium sulfinates was investigated (Table 2). A broad range of sodium benzenesulfinates bearing electron-donating groups $(\mathrm{Me}, \mathrm{MeO}$ and $\left.{ }^{t} \mathrm{Bu}\right)$ or electron-withdrawing groups $\left(\mathrm{F}, \mathrm{Cl}, \mathrm{Br}, \mathrm{CF}_{3}\right.$ and $\mathrm{NO}_{2}$ ) on the phenyl ring underwent the reaction efficiently, affording the corresponding $\mathrm{C}(4)$ sulfonylation products 
$\mathbf{3 b} \sim \mathbf{3 i}$ in high yields (Table 2). Moreover, the position of the substituent also had an effect on the reaction. Sodium benzenesulfinates that bear either electron-donating group (Me) or electron-withdrawing groups (F) at the paraposition of the phenyl ring were superior to their ortho-substituted counterparts in isolated yields (Table 2, $3 \mathbf{b}$ vs $\mathbf{3 j}$; 3e vs $\mathbf{3 k}$ ), which is probably due to the steric hindrance effect of ortho-position substitution. In addition, sodium naphthalene-2-sulfinate also proved to be suitable substrates, providing the corresponding products $\mathbf{3 1}$ in suitable yield. Apart from aromatic sodium sulfinates, less reactive aliphatic sodium sulfinates, such as sodium cyclopropanesulfinate, were tolerated in this transformation, affording product $\mathbf{3 m}$ in moderate yield.

Table 2 Substrate scope of sodium sulfonates $\mathbf{2}^{a, b}$
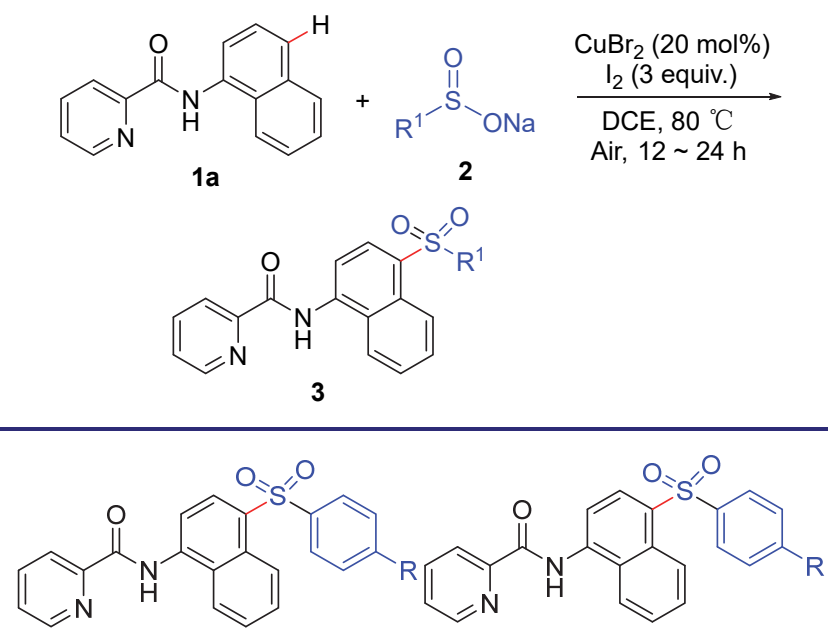

$$
\begin{array}{ll}
\text { 3a, } \mathrm{R}=\mathrm{H}, 90 \% & \mathbf{3 e}, \mathrm{R}=\mathrm{F}, 86 \% \\
\text { 3b, } \mathrm{R}=\mathrm{CH}_{3}, 85 \% & \mathbf{3 f}, \mathrm{R}=\mathrm{Cl}, 89 \% \\
\text { 3c, } \mathrm{R}=\mathrm{OCH}_{3}, 84 \% & \mathbf{3 g}, \mathrm{R}=\mathrm{Br}, 78 \% \\
\text { 3d, } \mathrm{R}={ }^{t} \mathrm{Bu}, 88 \% & \mathbf{3 h}, \mathrm{R}=\mathrm{CF}_{3}, 79 \% \\
& \mathbf{3 i}, \mathrm{R}=\mathrm{NO}_{2}, 65 \%
\end{array}
$$<smiles>[R]c1ccccc1S(=O)(=O)c1ccc(NC(=O)c2ccccn2)c2ccccc12</smiles><smiles>O=C(Nc1ccc(S(=O)(=O)C2CC2)c2ccccc12)c1ccccn1</smiles>

$3 \mathrm{~m}, 56 \%$

${ }^{a}$ Reaction conditions: 1a $(0.2 \mathrm{mmol}), 2(0.4 \mathrm{mmol}), \mathrm{CuBr}_{2}(0.04 \mathrm{mmol}, 0.2$ equiv.), $\mathrm{I}_{2}$ (0.6 mmol, 3.0 equiv.), DCE $(2 \mathrm{~mL})$, Air, $80{ }^{\circ} \mathrm{C}$, for $12 \sim 24 \mathrm{~h}$. ${ }^{b}$ Isolated yields.

Further application of this method focus on the substitued PA moiety, $N$-(naphthalen-1-yl)picolinamide with either electron-withdrawing groups, such as chlorine (3n), bromine (3o), trifluoromethyl (3q), ester (3p), or electron-donating groups, such as methyl $(\mathbf{3 r})$, on the pyridine ring of $\mathrm{N}$-(naphthalen-1-yl)picolinamide, were tolerated in this transformation, providing moderate to good yields (Table 3). Additionally, the isoquinoline-1-carboxamide as the directing group also gave the desired product in a moderate yield (3t). To our delight, 5,6,7,8-tetrahydro1 -naphthylamine could also tolerated in this transformation, and achieved the product in moderate yield (3s).

Table 3 Substrate scope of $N$-(naphthalen-1-yl)picolinamides $\mathbf{1}^{a, b}$

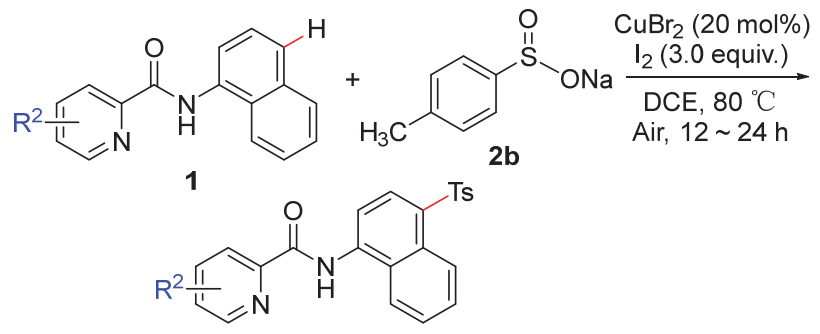

3<smiles>[R]c1ccc(C(=O)Nc2ccc([As])c3ccccc23)nc1</smiles>

$3 n, \mathrm{R}=\mathrm{Cl}, 83 \%$

3o, $\mathrm{R}=\mathrm{Br}, 75 \%$

$3 \mathbf{p}, \mathrm{R}=\mathrm{CO}_{2} \mathrm{CH}_{3}, 86 \%$

$3 \mathbf{q}, \mathrm{R}=\mathrm{CF}_{3}, 89 \%$<smiles>O=C(Nc1ccc([12F])c2c1CCCC2)c1ccccn1</smiles>

3s, $83 \%$<smiles>Cc1cccnc1C(=O)Nc1ccc([12F])c2ccccc12</smiles>

$3 \mathbf{r}, 78 \%$

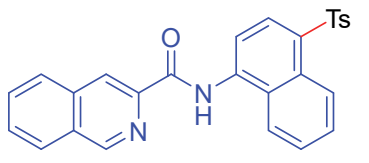

3t, $70 \%$
${ }^{a}$ Reaction conditions: 1a $(0.2 \mathrm{mmol}), 2(0.4 \mathrm{mmol}), \mathrm{CuBr}_{2}(0.04 \mathrm{mmol}, 0.2$ equiv.), $\mathrm{I}_{2}(0.6 \mathrm{mmol}, 3.0$ equiv. $)$, DCE $(2 \mathrm{~mL})$, air, $80{ }^{\circ} \mathrm{C}, 12 \sim 24 \mathrm{~h} .{ }^{b}$ Isolated yields.

The group of Willis and Georg have shown that lithium, manganese or zinc sulfinates, generated from the reaction of an organometallic reagent and an appropriate sulfur dioxide source are attractive and readily available alternatives to sodium sulfinates and suitable reagents for a variety of transformations. Therefore the reaction with lithium $p$-toluenesulfinate $\mathbf{4}$ as sulfonylation reagent was investigated in our transformation. The lithium salt was commercial available. To our delight, the copper-catalyzed reaction of the lithium ulfinate with $\mathrm{N}$-(naphthalen-1-yl)-picolinamide (1a), bearing the directing group, furnished the desired sulfone $\mathbf{3 b}$ in $85 \%$ yield (Scheme 2 ).

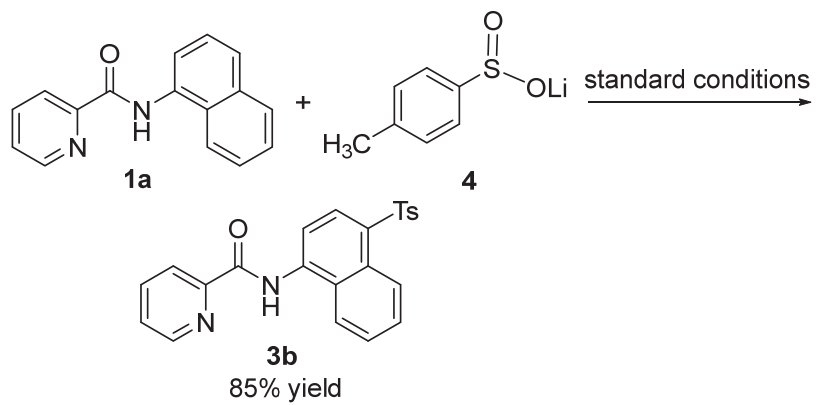

Scheme 2 Lithium $p$-toluenesulfinate as the sulfinate regant 
To demonstrate the synthetic of the new method, the reaction was carried out on a $4 \mathrm{mmol}$ gram scale, and the desired product was obtained in $82 \%$ yield (Scheme 3 ). The amide bond can be easily broken in $90 \%$ yield without damaging the $\mathrm{C}(4)$ sulfonylated product $\mathbf{3 b}$ to obtain $\mathbf{5}$.

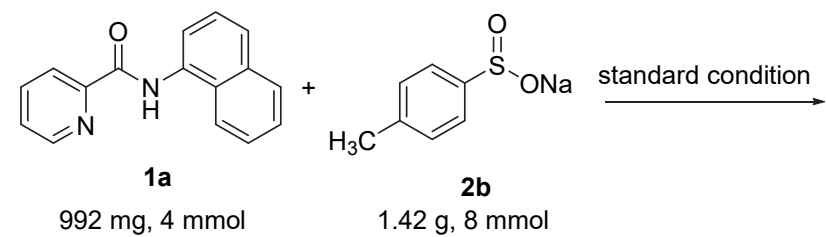<smiles>[3H]c1ccc(NC(=O)c2ccccn2)c2ccccc12</smiles>
$3 b$

$1.32 \mathrm{~g}, 82 \%$ yield<smiles>O=C(Nc1ccc(F)c2ccccc12)c1ccccn1</smiles>

$3 b$

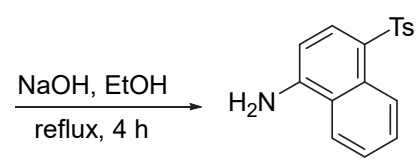

5, $90 \%$ yield
Scheme 3 Gram-scale C-H sulfonylation of 1a and removal of the directed group

To shed light on the possible pathway of this sulfonylation reaction, a number of control experiments were performed as depicted in Scheme 4. It was found that addition of 2,2,6,6-tetramethylpiperidin-1-yl)oxidanyl (TEMPO) and 2,6-di-tert-butyl-4-methylphenol (BHT) inhibited the reaction, and the yield was sharply decreased to $8 \%$ or trace (Scheme 4, a). Since TEMPO and BHT either scavenged radical intermediates or deactivated the copper catalyst or $I_{2}$ through redox processes or the formation of metal-complexes, this result proved the involvement of a single-electron-transfer (SET) processes and radical trap were conducted.

On the basis of the control experiments in Scheme 4 and relevant reports, ${ }^{[13,14,18]}$ a plausible mechanism as depicted in Scheme 5 was proposed. Firstly, copper salt $\mathrm{CuBr}_{2}$ and $N$-(naphthalen-1-yl)picolinamide (1a) gave aninicimidaatecopper(II) complex $\mathbf{A}$, and then complex A took place an intermolecular SET between the naphthalen-1-amine and either copper(II) or iodine furnishes the radical complex $\mathbf{B}$. Although both $\mathrm{Cu}^{\mathrm{II}}$ and $\mathrm{I}_{2}$ were competent oxidants for this reaction. Oxidation by $\mathrm{I}_{2}$ was more likely to occur, due to the higher oxidation potential of $\mathbf{I}_{\mathbf{2}}$. Single-electron oxidation of the sulfinate $\mathbf{2}$, most likely again with $\mathbf{I}_{2}$, leaded to the formation of sulfonyl radical $3{ }^{[19]}$ addition of the radical $\mathbf{3}$ to intermediate B formed complex C. Loss of proton, presumably via base-assisted deprotonation gave the anionic copper complex $\mathbf{D}$, which could undergo protonation to yield the desired product 3 as well as completion of the catalytic cycle. (a)

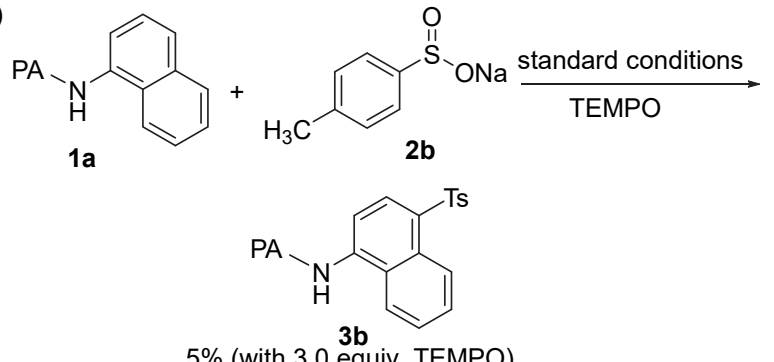

(b)

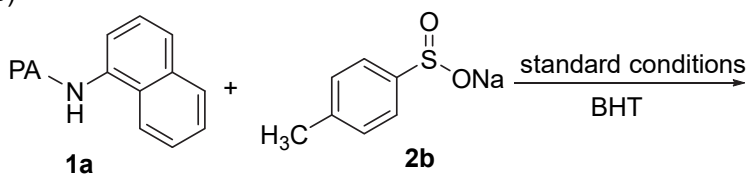<smiles>CCCNc1ccc([AsH3])c2ccccc12</smiles>

$8 \%$ (with 3.0 equiv. $\mathrm{BHT}$ )

Scheme 4 Control experiments

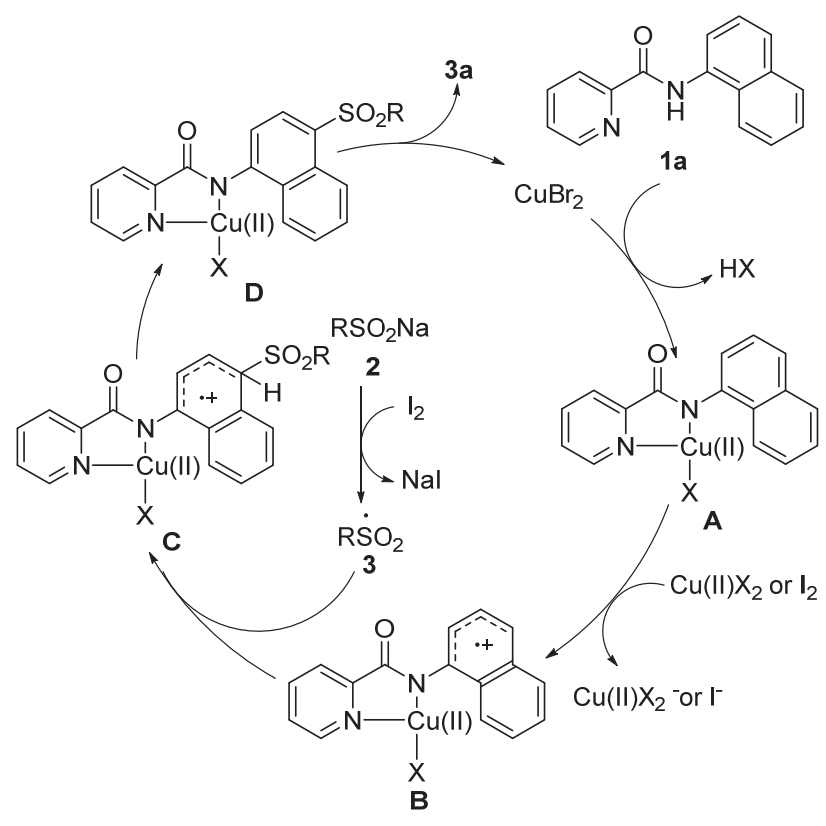

Scheme 5 Proposed mechanism

\section{Conclusions}

In summary, a novel, mild and efficient method for the remote sulfonylation of $N$-(naphthalen-1-yl)picolinamide with sodium sulfinates was developed. The coppercatalyzed reaction is simple to perform and proceeds readily under air. Various functional groups are tolerated and the desired $\mathrm{C}(4)$ sulfonylated $N$-(naphthalen-1-yl)picolinamide were obtained in high yields and regioselectivities. What is essential for the rapid $\mathrm{C}-\mathrm{H}$ functionalization with iodine is the co-oxidant. The facile iodine-mediated oxidant offers new opportunities for the development of novel 
mild $\mathrm{C}-\mathrm{H}$ activations.

\section{Experimental section}

\subsection{Reagents and solvents}

All starting materials were purchased from commerical sources and used without further purification. Solvents for column chromatography were technical standard. Chromatography column chromatography was performed with silica $200 \sim 400$ mesh. ${ }^{1} \mathrm{H}$ NMR, ${ }^{13} \mathrm{C}$ NMR and ${ }^{19} \mathrm{~F}$ NMR spectra were recorded on a Bruker Avance $400 \mathrm{MHz}$ spectrometer. Chemical shifts in ${ }^{1} \mathrm{H}$ NMR spectra are reported downfield from the internal standard $\mathrm{Me}_{4} \mathrm{Si}$ (TMS). Chemical shifts in ${ }^{13} \mathrm{C}$ NMR spectra are reported relative to the central line of the chloroform signal $(\delta$ 77.0). High resolution mass spectra were obtained with a Shimadzu LCMS-IT-TOF mass spectrometer. Analytical thin-layer chromatography (TLC) was performed using EM separations percolated silica gel $0.2 \mathrm{~mm}$ layer UV 254 fluorescent sheets.

\subsection{Typical procedure of the $\mathrm{Cu}(\mathrm{II})$-catalyzed cou- pling of sodium sulfinate}

An oven dried $10 \mathrm{~mL}$ tube was charged with a magnetic stirring bar, $\mathrm{N}$-(naphthalen-1-yl)picolinamide derivative $\mathbf{1}$ ( $0.2 \mathrm{mmol}, 1.0$ equiv.), sodium sulfonate 2 ( $0.4 \mathrm{mmol}, 2.0$ equiv.), $\mathrm{CuBr}_{2}$ ( $8.9 \mathrm{mg}, 0.04 \mathrm{mmol}, 0.2$ equiv.), $\mathrm{I}_{2}$ (152mg, $0.6 \mathrm{mmol}, 3.0$ equiv.) and DCE $(2 \mathrm{~mL})$. The reaction was stirred at $80{ }^{\circ} \mathrm{C}$ until the complete consumption of $\mathbf{1}$ as monitored by TLC analysis (typically $12 \sim 24 \mathrm{~h}$ ). The reaction mixture was then diluted with water and extracted with ethyl acetate. After the combined organic layers were washed with brine, dried over $\mathrm{Na}_{2} \mathrm{SO}_{4}$ and concentrated under reduced pressure, the residue was purified by flash column chromatography on silica gel to afford pure product 3.

$N$-(4-(Phenylsulfonyl)naphthalen-1-yl)picolinamide (3a): White solid, m.p. 193 $194{ }^{\circ} \mathrm{C}$; ${ }^{1} \mathrm{H}$ NMR $(400 \mathrm{MHz}$, $\left.\mathrm{CDCl}_{3}\right) \delta: 11.12(\mathrm{~s}, 1 \mathrm{H}), 8.77 \sim 8.67(\mathrm{~m}, 3 \mathrm{H}), 8.60(\mathrm{~d}, J=$ $8.3 \mathrm{~Hz}, 1 \mathrm{H}), 8.35(\mathrm{~d}, J=7.8 \mathrm{~Hz}, 1 \mathrm{H}), 8.19 \sim 8.12(\mathrm{~m}, 1 \mathrm{H})$, $7.96(\mathrm{~d}, J=4.0 \mathrm{~Hz}, 3 \mathrm{H}), 7.64$ (d, $J=12.8 \mathrm{~Hz}, 2 \mathrm{H}), 7.59 \sim$ $7.54(\mathrm{~m}, 1 \mathrm{H}), 7.48(\mathrm{~d}, J=14.6 \mathrm{~Hz}, 3 \mathrm{H}) ;{ }^{13} \mathrm{C}$ NMR $(101$ $\left.\mathrm{MHz}, \mathrm{CDCl}_{3}\right) \delta: 162.28,149.27,148.22,142.03,138.42$, $138.00,132.90,131.22,130.99$, 129.41, 129.06, 128.27, 127.25, 127.06, 125.97, 125.29, 122.69, 120.85, 115.18; HRMS (ESI) calcd for $\mathrm{C}_{22} \mathrm{H}_{17} \mathrm{~N}_{2} \mathrm{O}_{3} \mathrm{~S}[\mathrm{M}+\mathrm{H}]^{+} 389.0960$, found 389.0967.

$\mathrm{N}$-(4-Tosylnaphthalen-1-yl)picolinamide (3b): White solid, m.p. 231 232 ${ }^{\circ} \mathrm{C} ;{ }^{1} \mathrm{H}$ NMR $\left(400 \mathrm{MHz}, \mathrm{CDCl}_{3}\right) \delta$ : $11.04(\mathrm{~s}, 1 \mathrm{H}), 8.65(\mathrm{~s}, 3 \mathrm{H}), 8.51(\mathrm{~d}, J=7.0 \mathrm{~Hz}, 1 \mathrm{H}), 8.29$ $(\mathrm{d}, J=5.9 \mathrm{~Hz}, 1 \mathrm{H}), 8.13 \sim 8.03(\mathrm{~m}, 1 \mathrm{H}), 7.90(\mathrm{~s}, 1 \mathrm{H})$, $7.85 \sim 7.73(\mathrm{~m}, 2 \mathrm{H}), 7.56(\mathrm{~s}, 2 \mathrm{H}), 7.50(\mathrm{~s}, 1 \mathrm{H}), 7.19(\mathrm{~s}$, 2H), $2.28(\mathrm{~s}, 3 \mathrm{H}) ;{ }^{13} \mathrm{C}$ NMR $\left(101 \mathrm{MHz}, \mathrm{CDCl}_{3}\right) \delta: 162.28$, $149.33,148.23,143.80,139.09,138.23,137.98,131.47$, $130.90,129.69,129.38,128.18,127.35,127.02,126.00$, 125.38, 122.69, 120.81, 115.23, 21.50; HRMS (ESI) calcd for $\mathrm{C}_{23} \mathrm{H}_{19} \mathrm{~N}_{2} \mathrm{O}_{3} \mathrm{~S}[\mathrm{M}+\mathrm{H}]^{+}$403.1116, found 403.1119.
$N$-(4-((4-Methoxyphenyl)sulfonyl)naphthalen-1-yl)picolinamide (3c): White solid, m.p. $216 \sim 217{ }^{\circ} \mathrm{C} ;{ }^{1} \mathrm{H}$ NMR $\left(400 \mathrm{MHz}, \mathrm{CDCl}_{3}\right) \delta: 10.78(\mathrm{~s}, 1 \mathrm{H}), 8.71(\mathrm{~d}, J=4.3$ $\mathrm{Hz}, 1 \mathrm{H}), 8.48$ (d, J=7.9 Hz, 1H), 8.34 (d, $J=10.7 \mathrm{~Hz}$, $2 \mathrm{H}), 8.11(\mathrm{~d}, J=7.9 \mathrm{~Hz}, 1 \mathrm{H}), 7.94(\mathrm{~d}, J=7.7 \mathrm{~Hz}, 1 \mathrm{H})$, $7.65 \sim 7.57(\mathrm{~m}, 2 \mathrm{H}), 7.57 \sim 7.51(\mathrm{~m}, 2 \mathrm{H}), 7.30(\mathrm{~d}, J=8.8$ $\mathrm{Hz}, 2 \mathrm{H}), 6.87 \sim 6.80(\mathrm{~m}, 2 \mathrm{H}), 3.78(\mathrm{~s}, 3 \mathrm{H}) ;{ }^{13} \mathrm{C}$ NMR $(101$ $\left.\mathrm{MHz}, \mathrm{CDCl}_{3}\right) \delta: 162.21,159.03,149.84,148.14,137.77$, $133.17,132.70,132.31,130.40,130.08,126.95,126.67$, $126.62,126.19$, 126.05, 122.51, 120.91, 118.46, 114.91, 55.32; HRMS (ESI) calcd for $\mathrm{C}_{23} \mathrm{H}_{19} \mathrm{~N}_{2} \mathrm{O}_{4} \mathrm{~S}[\mathrm{M}+\mathrm{H}]^{+}$ 419.1066, found 419.0987 .

$N$-(4-((4-(tert-Butyl)phenyl)sulfonyl)naphthalen-1-yl)picolinamide (3d): White solid, m.p. 232 $233{ }^{\circ} \mathrm{C} ;{ }^{1} \mathrm{H}$ NMR (400 MHz, $\left.\mathrm{CDCl}_{3}\right) \delta: 11.10(\mathrm{~s}, 1 \mathrm{H}), 8.80(\mathrm{~d}, J=6.7$ $\mathrm{Hz}, 1 \mathrm{H}), 8.71(\mathrm{~d}, J=8.3 \mathrm{~Hz}, 2 \mathrm{H}), 8.58(\mathrm{~d}, J=8.3 \mathrm{~Hz}, 1 \mathrm{H})$, $8.36(\mathrm{~d}, J=7.7 \mathrm{~Hz}, 1 \mathrm{H}), 8.19 \sim 8.13(\mathrm{~m}, 1 \mathrm{H}), 7.97(\mathrm{t}, J=$ $7.6 \mathrm{~Hz}, 1 \mathrm{H}), 7.89(\mathrm{~d}, J=8.6 \mathrm{~Hz}, 2 \mathrm{H}), 7.67 \sim 7.62(\mathrm{~m}, 2 \mathrm{H})$, $7.59 \sim 7.53(\mathrm{~m}, 1 \mathrm{H}), 7.47(\mathrm{~d}, J=8.6 \mathrm{~Hz}, 2 \mathrm{H}), 1.27(\mathrm{~s}, 9 \mathrm{H})$; ${ }^{13} \mathrm{C}$ NMR $\left(101 \mathrm{MHz}, \mathrm{CDCl}_{3}\right) \delta: 162.27,156.76,149.38$, $148.22,139.05,138.24,138.00,131.65,131.04,129.53$, $128.19,127.17,127.01,126.10,125.53,122.72,120.83$, 115.36, 35.11, 30.98; HRMS (ESI) calcd for $\mathrm{C}_{26} \mathrm{H}_{25} \mathrm{~N}_{2} \mathrm{O}_{3} \mathrm{~S}$ $[\mathrm{M}+\mathrm{H}]^{+}$445.1586, found 445.1584.

$N$-(4-((4-Fluorophenyl)sulfonyl)naphthalen-1-yl)picolinamide (3e): White solid, m.p. $213 \sim 214{ }^{\circ} \mathrm{C}$; ${ }^{1} \mathrm{H}$ NMR $\left(400 \mathrm{MHz}, \mathrm{CDCl}_{3}\right) \delta: 11.15(\mathrm{~s}, 1 \mathrm{H}), 8.76 \sim 8.68(\mathrm{~m}, 3 \mathrm{H})$, $8.59(\mathrm{~d}, J=8.3 \mathrm{~Hz}, 1 \mathrm{H}), 8.38(\mathrm{~d}, \quad J=7.8 \mathrm{~Hz}, 1 \mathrm{H}), 8.21 \sim$ $8.15(\mathrm{~m}, 1 \mathrm{H}), 7.99(\mathrm{~d}, J=7.0 \mathrm{~Hz}, 3 \mathrm{H}), 7.71 \sim 7.61(\mathrm{~m}$, $2 \mathrm{H}), 7.58(\mathrm{~d}, J=7.5 \mathrm{~Hz}, 1 \mathrm{H}), 7.14(\mathrm{t}, J=8.5 \mathrm{~Hz}, 2 \mathrm{H}) ;{ }^{13} \mathrm{C}$ NMR (101 MHz, $\left.\mathrm{CDCl}_{3}\right) \delta: 166.43(\mathrm{~d}, J=254.0 \mathrm{~Hz})$, $163.89,162.30,149.29,148.23,138.59,138.21,138.18$, $138.00,131.24,130.86,130.11(\mathrm{~d}, J=9.3 \mathrm{~Hz}), 130.02$, $129.33,128.37,127.14,127.07,126.02,125.12,122.71$, $120.96,116.47,116.25$ (d, $J=22.6 \mathrm{~Hz}), 115.19 ;{ }^{19} \mathrm{~F}$ NMR $\left(376 \mathrm{MHz}, \mathrm{CDCl}_{3}\right) \delta$ : -104.64 ; HRMS (ESI) calcd for $\mathrm{C}_{22} \mathrm{H}_{16} \mathrm{FN}_{2} \mathrm{O}_{3} \mathrm{~S}[\mathrm{M}+\mathrm{H}]^{+}$407.0866, found 407.1409.

$\mathrm{N}$-(4-((4-Chlorophenyl)sulfonyl)naphthalen-1-yl)picolinamide (3f): White solid, m.p. $211 \sim 212{ }^{\circ} \mathrm{C}$; ${ }^{1} \mathrm{H}$ NMR $\left(400 \mathrm{MHz}, \mathrm{CDCl}_{3}\right) \delta: 11.14(\mathrm{~s}, 1 \mathrm{H}), 8.80 \sim 8.69(\mathrm{~m}, 2 \mathrm{H})$, $8.66(\mathrm{~d}, J=6.7 \mathrm{~Hz}, 1 \mathrm{H}), 8.59$ (d, $J=7.5 \mathrm{~Hz}, 1 \mathrm{H}), 8.35$ (d, $J=6.6 \mathrm{~Hz}, 1 \mathrm{H}), 8.16(\mathrm{~d}, J=6.0 \mathrm{~Hz}, 1 \mathrm{H}), 7.97(\mathrm{~s}, 1 \mathrm{H}), 7.89$ $(\mathrm{d}, J=7.0 \mathrm{~Hz}, 2 \mathrm{H}), 7.65(\mathrm{~s}, 2 \mathrm{H}), 7.57(\mathrm{~s}, 1 \mathrm{H}), 7.43(\mathrm{~d}, J=$ $6.9 \mathrm{~Hz}, 2 \mathrm{H}) ;{ }^{13} \mathrm{C} \mathrm{NMR}\left(101 \mathrm{MHz}, \mathrm{CDCl}_{3}\right) \delta: 162.30$, $149.20,148.24,140.55,139.49,138.68,138.01,131.40$, $130.41,129.38,129.29,128.71,128.44,127.17,127.10$, 125.93, 125.03, 122.70, 120.95, 115.10; HRMS (ESI) calcd for $\mathrm{C}_{22} \mathrm{H}_{16} \mathrm{ClN}_{2} \mathrm{O}_{3} \mathrm{~S}[\mathrm{M}+\mathrm{H}]^{+}$423.0570, found 423.0550 .

$N$-(4-((4-Bromophenyl)sulfonyl)naphthalen-1-yl)picolinamide (3g): White solid, m.p. $\left(225 \sim 226{ }^{\circ} \mathrm{C}\right.$; ${ }^{1} \mathrm{H}$ NMR $\left(400 \mathrm{MHz}, \mathrm{CDCl}_{3}\right) \delta: 11.15(\mathrm{~s}, 1 \mathrm{H}), 8.74(\mathrm{~d}, J=12.7 \mathrm{~Hz}$, $2 \mathrm{H}), 8.66(\mathrm{~d}, J=8.7 \mathrm{~Hz}, 1 \mathrm{H}), 8.59(\mathrm{~d}, J=8.3 \mathrm{~Hz}, 1 \mathrm{H}), 8.36$ $(\mathrm{d}, J=7.7 \mathrm{~Hz}, 1 \mathrm{H}), 8.17(\mathrm{~d}, J=7.7 \mathrm{~Hz}, 1 \mathrm{H}), 7.98(\mathrm{t}, J=$ $7.3 \mathrm{~Hz}, 1 \mathrm{H}), 7.82$ (d, $J=8.5 \mathrm{~Hz}, 2 \mathrm{H}), 7.66(\mathrm{~d}, J=6.9 \mathrm{~Hz}$, $2 \mathrm{H}), 7.58(\mathrm{t}, J=9.2 \mathrm{~Hz}, 3 \mathrm{H}) ;{ }^{13} \mathrm{C}$ NMR $\left(101 \mathrm{MHz}, \mathrm{CDCl}_{3}\right)$ $\delta: 162.30,149.24,148.25,141.13,138.71,138.02,132.37$, 
$131.45,130.37,129.32,128.79,128.46,128.05,127.19$, 127.11, 125.94, 125.06, 122.72, 120.96, 115.11; HRMS (ESI) calcd for $\mathrm{C}_{22} \mathrm{H}_{16} \mathrm{BrN}_{2} \mathrm{O}_{3} \mathrm{~S}[\mathrm{M}+\mathrm{H}]^{+} 467.0065$, found 467.0057.

$\mathrm{N}$-(4-((4-(Trifluoromethyl)phenyl)sulfonyl)naphthalen-1yl)picolinamide (3h): White solid, m.p. $229 \sim 230{ }^{\circ} \mathrm{C} ;{ }^{1} \mathrm{H}$ NMR $\left(400 \mathrm{MHz}, \mathrm{CDCl}_{3}\right) \delta: 11.17(\mathrm{~s}, 1 \mathrm{H}), 8.79$ (d, $J=8.4$ $\mathrm{Hz}, 1 \mathrm{H}), 8.73(\mathrm{~d}, J=4.2 \mathrm{~Hz}, 1 \mathrm{H}), 8.68(\mathrm{~d}, J=7.4 \mathrm{~Hz}, 1 \mathrm{H})$, $8.64(\mathrm{~d}, J=8.4 \mathrm{~Hz}, 1 \mathrm{H}), 8.37(\mathrm{~d}, J=7.8 \mathrm{~Hz}, 1 \mathrm{H}), 8.20 \sim$ $8.16(\mathrm{~m}, 1 \mathrm{H}), 8.09(\mathrm{~d}, J=8.2 \mathrm{~Hz}, 2 \mathrm{H}), 7.98(\mathrm{~d}, J=7.7 \mathrm{~Hz}$, $1 \mathrm{H}), 7.73(\mathrm{~d}, J=8.3 \mathrm{~Hz}, 2 \mathrm{H}), 7.69 \sim 7.64(\mathrm{~m}, 2 \mathrm{H}), 7.58(\mathrm{~d}$, $J=6.7 \mathrm{~Hz}, 1 \mathrm{H}) ;{ }^{13} \mathrm{C} \mathrm{NMR}\left(101 \mathrm{MHz}, \mathrm{CDCl}_{3}\right) \delta: 162.34$, $149.29,148.28,145.78,139.06,138.05,134.75,134.42$, $131.91,129.82,129.48,128.62,127.76,127.29,127.14$, $126.30,126.27,126.24$ (q, $J=3.3 \mathrm{~Hz}), 126.20,125.99$, $125.00,122.77$ (q, $J=271.5 \mathrm{~Hz}), 121.04,115.14 ;{ }^{19} \mathrm{~F}$ NMR (376 MHz, CDCl3) $\delta$ : -63.21 ; HRMS (ESI) calcd for $\mathrm{C}_{23} \mathrm{H}_{16} \mathrm{~F}_{3} \mathrm{~N}_{2} \mathrm{O}_{3} \mathrm{~S}[\mathrm{M}+\mathrm{H}]^{+}$457.0834, found 457.0828.

$\mathrm{N}$-(4-((4-Nitrophenyl)sulfonyl)naphthalen-1-yl)picolinamide (3i): White solid, m.p. $226 \sim 227{ }^{\circ} \mathrm{C}$; ${ }^{1} \mathrm{H}$ NMR $\left(400 \mathrm{MHz}, \mathrm{CDCl}_{3}\right) \delta: 11.19(\mathrm{~s}, 1 \mathrm{H}), 8.80(\mathrm{~d}, J=8.4 \mathrm{~Hz}$, $1 \mathrm{H}), 8.73(\mathrm{~d}, J=4.3 \mathrm{~Hz}, 1 \mathrm{H}), 8.64(\mathrm{~d}, J=8.4 \mathrm{~Hz}, 2 \mathrm{H}), 8.36$ $(\mathrm{d}, J=7.7 \mathrm{~Hz}, 1 \mathrm{H}), 8.29(\mathrm{~d}, J=8.7 \mathrm{~Hz}, 2 \mathrm{H}), 8.19(\mathrm{~d}, J=$ $7.8 \mathrm{~Hz}, 1 \mathrm{H}), 8.13(\mathrm{~d}, J=8.7 \mathrm{~Hz}, 2 \mathrm{H}), 7.99(\mathrm{t}, J=7.2 \mathrm{~Hz}$, $1 \mathrm{H}), 7.67(\mathrm{~d}, J=6.7 \mathrm{~Hz}, 2 \mathrm{H}), 7.61 \sim 7.55(\mathrm{~m}, 1 \mathrm{H}) ;{ }^{13} \mathrm{C}$ NMR $\left(101 \mathrm{MHz}, \mathrm{CDCl}_{3}\right) \delta: 162.35,150.15,149.20$, $148.28,147.89,139.37,138.07,132.21,129.43,129.14$, $128.79,128.49,127.39,127.19,125.94,124.76,124.32$, 122.78, 121.14, 115.10; HRMS (ESI) calcd for $\mathrm{C}_{22} \mathrm{H}_{16^{-}}$ $\mathrm{N}_{2} \mathrm{O}_{3} \mathrm{~S}[\mathrm{M}+\mathrm{H}]^{+}$434.0811, found 434.0853.

$\mathrm{N}$-(4-(o-Tolylsulfonyl)naphthalen-1-yl)picolinamide (3j): White solid, m.p. $210 \sim 211{ }^{\circ} \mathrm{C}$; ${ }^{1} \mathrm{H}$ NMR $(400 \mathrm{MHz}$, $\left.\mathrm{CDCl}_{3}\right) \delta: 11.14(\mathrm{~s}, 1 \mathrm{H}), 8.76(\mathrm{~d}, J=8.4 \mathrm{~Hz}, 1 \mathrm{H}), 8.72(\mathrm{~d}$, $J=4.0 \mathrm{~Hz}, 1 \mathrm{H}), 8.58 \sim 8.51(\mathrm{~m}, 2 \mathrm{H}), 8.36(\mathrm{~d}, J=7.8 \mathrm{~Hz}$, $1 \mathrm{H}), 8.33 \sim 8.28(\mathrm{~m}, 1 \mathrm{H}), 8.17(\mathrm{~d}, J=8.4 \mathrm{~Hz}, 1 \mathrm{H}), 7.97(\mathrm{t}$, $J=8.3 \mathrm{~Hz}, 1 \mathrm{H}), 7.64(\mathrm{t}, J=7.5 \mathrm{~Hz}, 1 \mathrm{H}), 7.6 \sim 7.54(\mathrm{~m}$, 2H), $7.42(\mathrm{t}, J=7.3 \mathrm{~Hz}, 2 \mathrm{H}), 7.18(\mathrm{~d}, J=6.8 \mathrm{~Hz}, 1 \mathrm{H})$, $2.41(\mathrm{~s}, 3 \mathrm{H}) ;{ }^{13} \mathrm{C} \mathrm{NMR}\left(101 \mathrm{MHz}, \mathrm{CDCl}_{3}\right) \delta$ : 162.29, $149.41,148.25,139.91,138.31,137.99,133.25,132.78$, $131.59,130.94,129.41,129.10,128.09,127.03,126.33$, $125.89,125.31,122.71,120.80,114.80,20.13$; HRMS (ESI) calcd for $\mathrm{C}_{23} \mathrm{H}_{19} \mathrm{~N}_{2} \mathrm{O}_{3} \mathrm{~S}[\mathrm{M}+\mathrm{H}]^{+}$403.1116, found 403.1119 .

$N$-(4-((2-Chlorophenyl)sulfonyl)naphthalen-1-yl)picolinamide (3k): White solid, m.p. $211 \sim 212{ }^{\circ} \mathrm{C}$; ${ }^{1} \mathrm{H}$ NMR $\left(400 \mathrm{MHz}, \mathrm{CDCl}_{3}\right) \delta: 11.17(\mathrm{~s}, 1 \mathrm{H}), 8.80(\mathrm{~d}, J=8.4 \mathrm{~Hz}$, $1 \mathrm{H}), 8.71(\mathrm{~d}, J=8.5 \mathrm{~Hz}, 2 \mathrm{H}), 8.54$ (d, $J=7.7 \mathrm{~Hz}, 1 \mathrm{H}), 8.46$ $(\mathrm{d}, J=8.5 \mathrm{~Hz}, 1 \mathrm{H}), 8.36(\mathrm{~d}, J=7.7 \mathrm{~Hz}, 1 \mathrm{H}), 8.17(\mathrm{~d}, J=$ $8.4 \mathrm{~Hz}, 1 \mathrm{H}), 7.97(\mathrm{t}, J=7.6 \mathrm{~Hz}, 1 \mathrm{H}), 7.66 \sim 7.60(\mathrm{~m}, 1 \mathrm{H})$, $7.55(\mathrm{dd}, J=16.4,8.1 \mathrm{~Hz}, 3 \mathrm{H}), 7.48(\mathrm{t}, J=7.6 \mathrm{~Hz}, 1 \mathrm{H})$, $7.35(\mathrm{~d}, J=7.7 \mathrm{~Hz}, 1 \mathrm{H}) ;{ }^{13} \mathrm{C} \mathrm{NMR}\left(101 \mathrm{MHz}, \mathrm{CDCl}_{3}\right) \delta$ : $162.25,149.29,148.22,139.14,138.66,138.00,134.34$, $133.65,133.01,132.18,130.87,129.34,129.29,128.18$, $127.14,127.06,126.87,125.61,124.72,122.69,120.93$, 114.67; HRMS (ESI) calcd for $\mathrm{C}_{22} \mathrm{H}_{16} \mathrm{ClN}_{2} \mathrm{O}_{3} \mathrm{~S}[\mathrm{M}+\mathrm{H}]^{+}$ 423.0570, found 423.0536 .

N-(4-(Naphthalen-2-ylsulfonyl)naphthalen-1-yl)picol- inamide (3l): White solid, m.p. $218 \sim 219{ }^{\circ} \mathrm{C}$; ${ }^{1} \mathrm{H}$ NMR $\left(400 \mathrm{MHz}, \mathrm{CDCl}_{3}\right) \delta: 11.13(\mathrm{~s}, 1 \mathrm{H}), 8.82 \sim 8.75(\mathrm{~m}, 2 \mathrm{H})$, $8.73 \sim 8.64(\mathrm{~m}, 3 \mathrm{H}), 8.36(\mathrm{~d}, J=7.8 \mathrm{~Hz}, 1 \mathrm{H}), 8.18 \sim 8.11$ (m, 1H), $7.97(\mathrm{t}, J=7.8 \mathrm{~Hz}, 2 \mathrm{H}), 7.89 \sim 7.80(\mathrm{~m}, 3 \mathrm{H}), 7.59$ $(\mathrm{t}, J=12.9 \mathrm{~Hz}, 5 \mathrm{H}) ;{ }^{13} \mathrm{C} \mathrm{NMR}\left(101 \mathrm{MHz}, \mathrm{CDCl}_{3}\right) \delta$ : $162.30,149.31,148.23,138.95,138.47,137.99,134.87$, $132.09,131.37,130.95,129.44,129.41,129.36,128.96$, $128.53,128.30,127.87,127.51,127.05,125.98,125.31$, 122.70, 122.48, 120.82, 115.19; HRMS (ESI) calcd for $\mathrm{C}_{26} \mathrm{H}_{19} \mathrm{~N}_{2} \mathrm{O}_{3} \mathrm{~S}[\mathrm{M}+\mathrm{H}]^{+}$439.1116, found 439.1066.

$N$-(4-(Cyclopropylsulfonyl)naphthalen-1-yl)picolinamide (3m): White solid, m.p. $197 \sim 198{ }^{\circ} \mathrm{C} ;{ }^{1} \mathrm{H}$ NMR (400 $\left.\mathrm{MHz}, \mathrm{CDCl}_{3}\right) \delta: 11.12(\mathrm{~s}, 1 \mathrm{H}), 8.98 \sim 8.92(\mathrm{~m}, 1 \mathrm{H}), 8.72$ $(\mathrm{d}, J=4.5 \mathrm{~Hz}, 1 \mathrm{H}), 8.65(\mathrm{~d}, J=8.3 \mathrm{~Hz}, 1 \mathrm{H}), 8.34$ (d, $J=$ $7.8 \mathrm{~Hz}, 1 \mathrm{H}), 8.28$ (d, $J=8.3 \mathrm{~Hz}, 1 \mathrm{H}), 8.24 \sim 8.19(\mathrm{~m}, 1 \mathrm{H})$, $8.00 \sim 7.94(\mathrm{~m}, 1 \mathrm{H}), 7.74(\mathrm{p}, J=6.6 \mathrm{~Hz}, 2 \mathrm{H}), 7.60 \sim 7.54$ $(\mathrm{m}, 1 \mathrm{H}), 2.75$ (t, $J=8.1 \mathrm{~Hz}, 1 \mathrm{H}), 1.38(\mathrm{~d}, J=6.6 \mathrm{~Hz}, 2 \mathrm{H})$, $1.05 \sim 0.96(\mathrm{~m}, 2 \mathrm{H}) ;{ }^{13} \mathrm{C}$ NMR $\left(101 \mathrm{MHz}, \mathrm{CDCl}_{3}\right) \delta$ : $162.31,149.28,148.23,137.97,131.30,130.37,129.83$, $128.33,127.11,127.04,126.12,125.41,122.65,121.04$, 115.36, 33.26, 6.20; HRMS (ESI) calcd for $\mathrm{C}_{19} \mathrm{H}_{17} \mathrm{~N}_{2} \mathrm{O}_{3} \mathrm{~S}$ $[\mathrm{M}+\mathrm{H}]^{+}$353.0960, found 353.0958.

5-Chloro- $N$-(4-tosylnaphthalen-1-yl)picolinamide (3n): ${ }^{1} \mathrm{H}$ NMR (400 MHz, $\left.\mathrm{CDCl}_{3}\right) \delta$ : White solid, m.p. 209 $210{ }^{\circ} \mathrm{C} ; 10.86(\mathrm{~s}, 1 \mathrm{H}), 8.75$ (d, $\left.J=7.3 \mathrm{~Hz}, 1 \mathrm{H}\right), 8.69$ (d, $J=8.2 \mathrm{~Hz}, 2 \mathrm{H}), 8.58$ (d, $J=8.3 \mathrm{~Hz}, 1 \mathrm{H}), 8.32$ (d, $J=8.3$ $\mathrm{Hz}, 1 \mathrm{H}), 8.11$ (d, $J=7.0 \mathrm{~Hz}, 1 \mathrm{H}), 7.96(\mathrm{~d}, J=8.2 \mathrm{~Hz}, 1 \mathrm{H})$, $7.86(\mathrm{~d}, J=8.1 \mathrm{~Hz}, 2 \mathrm{H}), 7.65(\mathrm{~d}, J=7.2 \mathrm{~Hz}, 2 \mathrm{H}), 7.28$ (s, 2H), 2.37 (s, 3H); $\left.{ }^{13} \mathrm{C} \mathrm{NMR} \mathrm{(101} \mathrm{MHz,} \mathrm{CDCl}_{3}\right) \delta: 161.42$, $147.47,147.33,143.86,139.02,137.91,137.72,135.99$, $131.83,130.85,129.70,129.37,128.24,127.37,127.12$, $126.00,125.44,123.71,120.67,115.47,21.50$; HRMS (ESI) calcd for $\mathrm{C}_{23} \mathrm{H}_{18} \mathrm{ClN}_{2} \mathrm{O}_{3} \mathrm{~S} \quad[\mathrm{M}+\mathrm{H}]^{+}$437.0727, found 437.0673 .

5-Bromo- $N$-(4-tosylnaphthalen-1-yl)picolinamide (3o): White solid, m.p. $218 \sim 220{ }^{\circ} \mathrm{C}$; ${ }^{1} \mathrm{H}$ NMR $(400 \mathrm{MHz}$, $\left.\mathrm{CDCl}_{3}\right) \delta: 10.86(\mathrm{~s}, 1 \mathrm{H}), 8.78(\mathrm{~s}, 1 \mathrm{H}), 8.75(\mathrm{~d}, J=6.5 \mathrm{~Hz}$, $1 \mathrm{H}), 8.68(\mathrm{~d}, J=8.3 \mathrm{~Hz}, 1 \mathrm{H}), 8.58(\mathrm{~d}, J=8.3 \mathrm{~Hz}, 1 \mathrm{H})$, $8.26(\mathrm{~d}, J=8.3 \mathrm{~Hz}, 1 \mathrm{H}), 8.15 \sim 8.07(\mathrm{~m}, 2 \mathrm{H}), 7.86(\mathrm{~d}, J=$ $8.2 \mathrm{~Hz}, 2 \mathrm{H}), 7.65(\mathrm{~d}, J=5.6 \mathrm{~Hz}, 2 \mathrm{H}), 2.37(\mathrm{~s}, 3 \mathrm{H}) ;{ }^{13} \mathrm{C}$ NMR $\left(101 \mathrm{MHz}, \mathrm{CDCl}_{3}\right) \delta: 161.57,149.53,147.82$, $143.86,140.69,139.01,137.88,131.84,130.85,129.71$, $129.37,128.24,127.37,127.12,126.00,125.45,124.97$, 124.07, 120.66, 115.49, 21.51; HRMS (ESI) calcd for $\mathrm{C}_{23} \mathrm{H}_{28} \mathrm{BrN}_{2} \mathrm{O}_{3} \mathrm{~S}[\mathrm{M}+\mathrm{H}]^{+}$480.0143, found 481.0161.

5-(Methoxycarbonyl)- $N$-(4-tosylnaphthalen-1-yl)picolinamide (3p): White solid, m.p. 206 207 ${ }^{\circ} \mathrm{C}$; ${ }^{1} \mathrm{H}$ NMR $\left(400 \mathrm{MHz}, \mathrm{CDCl}_{3}\right) \delta: 11.03(\mathrm{~s}, 1 \mathrm{H}), 9.30(\mathrm{~s}, 1 \mathrm{H}), 8.78 \sim$ $8.66(\mathrm{~m}, 2 \mathrm{H}), 8.61 \sim 8.52(\mathrm{~m}, 2 \mathrm{H}), 8.44(\mathrm{~d}, J=8.1 \mathrm{~Hz}$, $1 \mathrm{H}), 8.13(\mathrm{~d}, J=7.6 \mathrm{~Hz}, 1 \mathrm{H}), 7.84(\mathrm{~d}, J=8.2 \mathrm{~Hz}, 2 \mathrm{H}), 7.64$ $(\mathrm{d}, J=6.6 \mathrm{~Hz}, 2 \mathrm{H}), 7.25(\mathrm{~s}, 2 \mathrm{H}), 4.02(\mathrm{~s}, 3 \mathrm{H}), 2.35$ (s, 3H); ${ }^{13} \mathrm{C}$ NMR $\left(101 \mathrm{MHz}, \mathrm{CDCl}_{3}\right) \delta: 164.77,161.30,152.15$, $149.48,143.88,139.18,138.94,137.81,131.92,130.81$, $129.71,129.33,128.81,128.27,127.36,127.17,125.99$, 125.42, 122.34, 120.69, 115.50, 52.84, 21.50; HRMS (ESI) calcd for $\mathrm{C}_{25} \mathrm{H}_{21} \mathrm{~N}_{2} \mathrm{O}_{5} \mathrm{~S}[\mathrm{M}+\mathrm{H}]^{+}$461.1171, found 461.1172 . 
5-Trifluoromethyl- $N$-(4-tosylnaphthalen-1-yl)picolinamide (3q): White solid, m.p. 219 $220{ }^{\circ} \mathrm{C} ;{ }^{1} \mathrm{H}$ NMR (400 $\left.\mathrm{MHz}, \mathrm{CDCl}_{3}\right) \delta: 10.94(\mathrm{~s}, 1 \mathrm{H}), 9.00(\mathrm{~s}, 1 \mathrm{H}), 8.74(\mathrm{~d}, J=$ $6.8 \mathrm{~Hz}, 1 \mathrm{H}), 8.68(\mathrm{~d}, J=8.3 \mathrm{~Hz}, 1 \mathrm{H}), 8.58(\mathrm{~d}, J=8.3 \mathrm{~Hz}$, $1 \mathrm{H}), 8.50(\mathrm{~d}, J=8.2 \mathrm{~Hz}, 1 \mathrm{H}), 8.26 \sim 8.21(\mathrm{~m}, 1 \mathrm{H}), 8.10(\mathrm{~d}$, $J=6.8 \mathrm{~Hz}, 1 \mathrm{H}), 7.86(\mathrm{~s}, 1 \mathrm{H}), 7.84(\mathrm{~s}, 1 \mathrm{H}), 7.67 \sim 7.60(\mathrm{~m}$, 2H), $7.26(\mathrm{~d}, J=8.1 \mathrm{~Hz}, 2 \mathrm{H}), 2.35(\mathrm{~s}, 3 \mathrm{H}) ;{ }^{13} \mathrm{C}$ NMR $(101$ $\left.\mathrm{MHz}, \mathrm{CDCl}_{3}\right) \delta: 160.83,152.20,145.47,145.43,145.39$, $145.35,143.92,138.91,137.61,135.54,135.51,135.48$, $135.45,132.17,130.75,129.72,129.34,128.31,127.38$ (q, $J=3.3 \mathrm{~Hz}), 127.23,126.02,122.63$ (q, $J=271.5 \mathrm{~Hz})$, 120.59, 115.67, 21.50; ${ }^{19} \mathrm{~F}$ NMR (376 MHz, $\left.\mathrm{CDCl}_{3}\right) \delta:-$ 62.49; HRMS (ESI) calcd for $\mathrm{C}_{24} \mathrm{H}_{18} \mathrm{~F}_{3} \mathrm{~N}_{2} \mathrm{O}_{3} \mathrm{~S}[\mathrm{M}+\mathrm{H}]^{+}$ 471.0990, found 471.0970.

3-Methyl- $N$-(4-tosylnaphthalen-1-yl)picolinamide (3r): White solid, m.p. 206 $207{ }^{\circ} \mathrm{C}$; ${ }^{1} \mathrm{H}$ NMR $(400 \mathrm{MHz}$, $\left.\mathrm{CDCl}_{3}\right) \delta: 11.44(\mathrm{~s}, 1 \mathrm{H}), 8.76 \sim 8.68(\mathrm{~m}, 2 \mathrm{H}), 8.59(\mathrm{~d}, J=$ $8.5 \mathrm{~Hz}, 2 \mathrm{H}), 8.15(\mathrm{~d}, J=7.7 \mathrm{~Hz}, 1 \mathrm{H}), 7.87$ (d, $J=8.2 \mathrm{~Hz}$, 2H), $7.72(\mathrm{~d}, J=7.4 \mathrm{~Hz}, 1 \mathrm{H}), 7.67 \sim 7.59(\mathrm{~m}, 2 \mathrm{H}), 7.50 \sim$ $7.43(\mathrm{~m}, 1 \mathrm{H}), 7.31 \sim 7.26(\mathrm{~m}, 2 \mathrm{H}), 2.88(\mathrm{~s}, 3 \mathrm{H}), 2.37(\mathrm{~s}$, $3 \mathrm{H}) ;{ }^{13} \mathrm{C} \mathrm{NMR}\left(101 \mathrm{MHz}, \mathrm{CDCl}_{3}\right) \delta: 163.68,146.14$, $145.56,143.73,141.59,139.19,138.72,136.76,131.02$, $129.66,129.41,128.09,127.31,126.87,126.61,126.14$, 125.29, 121.03, 114.89, 21.49, 20.81; HRMS (ESI) calcd for $\mathrm{C}_{24} \mathrm{H}_{21} \mathrm{~N}_{2} \mathrm{O}_{3} \mathrm{~S}[\mathrm{M}+\mathrm{H}]^{+}$417.1273, found 417.1279.

$N$-(4-Tosyl-5,6,7,8-tetrahydronaphthalen-1-yl)picolinamide (3s): White solid, m.p. $216 \sim 217{ }^{\circ} \mathrm{C}$; ${ }^{1} \mathrm{H}$ NMR (400 $\left.\mathrm{MHz}, \mathrm{CDCl}_{3}\right) \delta: 10.34(\mathrm{~s}, 1 \mathrm{H}), 8.62(\mathrm{~d}, J=4.1 \mathrm{~Hz}, 1 \mathrm{H})$, $8.50(\mathrm{~d}, J=8.8 \mathrm{~Hz}, 1 \mathrm{H}), 8.30(\mathrm{~d}, J=7.8 \mathrm{~Hz}, 1 \mathrm{H}), 8.17$ (d, $J=8.8 \mathrm{~Hz}, 1 \mathrm{H}), 7.93(\mathrm{t}, J=7.4 \mathrm{~Hz}, 1 \mathrm{H}), 7.73(\mathrm{~d}, J=8.0$ $\mathrm{Hz}, 2 \mathrm{H}), 7.50$ (d, $J=5.4 \mathrm{~Hz}, 1 \mathrm{H}), 7.28$ (d, $J=8.0 \mathrm{~Hz}, 2 \mathrm{H})$, $2.91(\mathrm{t}, J=5.9 \mathrm{~Hz}, 2 \mathrm{H}), 2.77(\mathrm{t}, J=6.1 \mathrm{~Hz}, 2 \mathrm{H}), 2.41(\mathrm{~s}$, $3 \mathrm{H}), 1.86 \sim 1.78(\mathrm{~m}, 2 \mathrm{H}), 1.72 \sim 1.67(\mathrm{~m}, 2 \mathrm{H}) ;{ }^{13} \mathrm{C} \mathrm{NMR}$ $\left(101 \mathrm{MHz}, \mathrm{CDCl}_{3}\right) \delta: 162.06,149.54,148.13,143.63$, $140.45,138.71,137.97,137.84,133.92,129.56,128.50$, $127.93,127.54,126.82,122.54,117.10,26.67,24.87$, 21.67, 21.56; HRMS (ESI) calcd for $\mathrm{C}_{23} \mathrm{H}_{123} \mathrm{~N}_{2} \mathrm{O}_{3} \mathrm{~S}[\mathrm{M}+$ $\mathrm{H}]^{+}$407.1429, found 407.1499.

$\mathrm{N}$-(4-Tosylnaphthalen-1-yl)quinoline-2-carboxamide (3t): White solid, m.p. $208 \sim 219{ }^{\circ} \mathrm{C}$; ${ }^{1} \mathrm{H}$ NMR $(400 \mathrm{MHz}$, $\left.\mathrm{CDCl}_{3}\right) \delta: 11.55(\mathrm{~s}, 1 \mathrm{H}), 9.79(\mathrm{~d}, J=7.0 \mathrm{~Hz}, 1 \mathrm{H}), 8.75(\mathrm{~d}$, $J=8.2 \mathrm{~Hz}, 2 \mathrm{H}), 8.62(\mathrm{t}, J=6.2 \mathrm{~Hz}, 2 \mathrm{H}), 8.19$ (d, $J=7.9$ $\mathrm{Hz}, 1 \mathrm{H}), 7.96 \sim 7.90(\mathrm{~m}, 2 \mathrm{H}), 7.88(\mathrm{~d}, J=8.2 \mathrm{~Hz}, 2 \mathrm{H})$, $7.83 \sim 7.75(\mathrm{~m}, 2 \mathrm{H}), 7.64(\mathrm{~d}, J=8.2 \mathrm{~Hz}, 2 \mathrm{H}), 7.30 \sim 7.26$ $(\mathrm{m}, 2 \mathrm{H}), 2.37(\mathrm{~s}, 3 \mathrm{H}) ;{ }^{13} \mathrm{C} \mathrm{NMR}\left(101 \mathrm{MHz}, \mathrm{CDCl}_{3}\right) \delta$ : $163.76,146.64,143.76,140.00,139.23,138.62,137.76$, $131.36,130.98,130.88,129.68,129.47,129.35,128.14$, $127.55,127.43,127.35,127.07,126.96,126.32,125.58$, 125.36, 121.08, 115.25, 21.48; HRMS (ESI) calcd for $\mathrm{C}_{27} \mathrm{H}_{21} \mathrm{~N}_{2} \mathrm{O}_{3} \mathrm{~S}[\mathrm{M}+\mathrm{H}]^{+}$453.1273, found 453.1274.

\subsection{Control experiments procedure}

To a $5 \mathrm{~mL}$ tube equipped with a magnetic stirring bar, $N$-(1-naphthyl)picolinamide $1(49.6 \mathrm{mg}, 0.2 \mathrm{mmol})$, sodium sulfinate (2b) $(71.2 \mathrm{mg}, 0.4 \mathrm{mmol}), \mathrm{CuBr}_{2}(8.9 \mathrm{mg}$, $0.04 \mathrm{mmol}$ ), 2,2,6,6-tetramethylpiperidineoxy (TEMPO, $93.7 \mathrm{mg}, 0.6 \mathrm{mmol})$ and DCE $(2 \mathrm{~mL})$ were added. No spe- cial precautions were taken to exclude moisture and air. The reaction was stirred at $80{ }^{\circ} \mathrm{C}$ for $12 \mathrm{~h}$. The reaction mixture was then diluted with water and extracted with ethyl acetate. After the combined organic layers were washed with brine, dried over $\mathrm{Na}_{2} \mathrm{SO}_{4}$ and concentrated under reduced pressure, the residue was purified by flash column chromatography on silica gel to afford the pure product 3a (4.1 mg, 5\% yield).

To a $5 \mathrm{~mL}$ tube equipped with a magnetic stirring bar, $N$-(1-naphthyl)picolinamide $1(49.6 \mathrm{mg}, 0.2 \mathrm{mmol})$, sodium sulfinate (2b) $(71.2 \mathrm{mg}, 0.4 \mathrm{mmol}), \mathrm{CuBr}_{2}(8.9 \mathrm{mg}$, $0.04 \mathrm{mmol}$ ), butylated hydroxytoluene (BHT, $132 \mathrm{mg}, 0.6$ $\mathrm{mmol})$ and DCE $(2 \mathrm{~mL})$ were added. No special precautions were taken to exclude moisture and air. The reaction was stirred at $80{ }^{\circ} \mathrm{C}$ for $12 \mathrm{~h}$. The reaction mixture was then diluted with water and extracted with ethyl acetate. After the combined organic layers were washed with brine, dried over $\mathrm{Na}_{2} \mathrm{SO}_{4}$, and concentrated under reduced pressure, the residue was purified by flash column chromatography on silica gel to afford the pure product $3 \mathbf{a}(6.4 \mathrm{mg}$, $8 \%$ yield).

Supporting Information ${ }^{1} \mathrm{H}$ NMR, ${ }^{13} \mathrm{C}$ NMR and ${ }^{19} \mathrm{~F}$ NMR spectra of compounds 3. The Supporting Information is available free of charge via the Internet at http://siocjournal.cn.

\section{References}

[1] (a) Patai, S.; Rappoport, C. Z.; Stirling, J. M. The Chemistry of Sulfones and Sulfoxides, Wiley, New York, 1988.

(b) Simpkins, N. S. Sulphones in Organic Synthesis, Pergamon Press, Oxford, 1993.

[2] La Regina, G.; Coluccia, A.; Brancale, A.; Piscitelli, F.; Gatti, V.; Maga, G.; Samuele, A.; Pannecouque, C.; Schols, D.; Balzarini, J.; Novellino E.; Silvestri, R. J. Med. Chem. 2011, 54, 1587.

[3] Ivachtchenko, A. V.; Golovina, E. S.; Kadieva, M. G.; Kysil, V. M.; Mitkin, O. D.; Tkachenko, S. E.; Okun, I. M. J. Med. Chem. 2011, 54,8161 .

[4] (a) Catarinella, M.; Gruner, T.; Strittmatter, T.; Marx, A.; Mayer, T. U. Angew. Chem., Int. Ed. 2009, 48, 9072.

(b) Lu, Q.; Zhang, J.; Wei, F.; Qi, Y.; Wang, H.; Liu, Z.; Lei, A. Angew. Chem., Int. Ed. 2013, 52, 7156.

(c) Lu, Q.; Zhang, J.; Zhao, G.; Qi, Y.; Wang, H.; Lei, A. J. Am. Chem. Soc. 2013, 135, 11481.

(d) Reddy, M. A.; Reddy, P. S.; Sreedhar, B. Adv. Synth. Catal. 2010, 352, 1861 .

(e) Fang, X.; Wang, W.; Yang, X.-Y.; Wu, F.-H. Chin. J. Org. Chem. 2021, 41, 412 (in Chinese).

(方向，王旺，杨雪艳，吴范宏，有机化学, 2021, 41, 412.)

[5] (a) Du, B.; Qian, P.; Wang, Y.; Mei, H.; Han, J.; Pan, Y. Org. Lett. 2016, $18,4144$.

(b) Jiang, Q.; Xu, B.; Jia, J.; Zhao, A.; Zhao, Y. R.; Li, Y. Y.; He, N. N.; Guo, C.C. J. Org. Chem. 2014, 79, 7372.

(c) Li, J.-M.; Weng, J.; Lu, G.; Chan, A. S. C. Tetrahedron Lett. 2016, 57, 212.

(d) Liang, S.; Manolikakes, G. Adv. Synth. Catal. 2016, 358, 2371.

(e) Liu, X.; Chen, X.; Mohr, J. T. Org. Lett. 2015, 17, 3572.

(f) Qiao, H.; Sun, S.; Yang, F.; Zhu, Y.; Zhu, W.; Dong, Y.; Wu, Y.; Kong, X.; Jiang, L.; Wu, Y. Org. Lett. 2015, 17, 6086.

(g) Rao, W. H.; Shi, B. F. Org. Lett. 2015, 17, 2784.

(h) Rokade, B. V.; Prabhu, K. R. J. Org. Chem. 2014, 79, 8110.

(i) Liu, D.; Mei, T.-S. Chin. J. Org. Chem. 2019, 39, 3600 (in Chi- 
nese).

(刘冬, 梅天胜, 有机化学, 2019, 39, 3600.)

[6] (a) Liu, N. M.; Liang, S.; Manolikakes, G. Synthesis 2016, 48, 1939.

(b) Shen, C.; Zhang, P. F.; Sun, Q.; Bai, S. Q.; Hor, T. S. A.; Liu, X. G. Chem. Soc. Rev. 2015, 44, 291.

(c) Shaaban, S.; Liang, S.; Liu, N. W.; Manolikakes, G. Org. Biomol. Chem. 2017, 15, 1947.

[7] (a) Zhao, X. D.; Dimitrijevic, E.; Dong, V. M. J. Am. Chem. Soc. 2009, 131, 3466.

(b) Liu, J. D.; Yu, L.; Zhuang, S. B.; Gui, Q. W.; Chen, X.; Wang, W. D.; Tan, Z. Chem. Commun. 2015, 51, 6418.

(c) Liang, H. W.; Jiang, K.; Ding, W.; Yuan, Y.; Shuai, L.; Chen, Y. C.; Wei, Y. Chem. Commun. 2015, 51, 16928.

(g) Xia, C. C.; Wang, K.; Xu, J.; Wei, Z. J.; Shen, C.; Duan, G. Y.; Zhu, Q.; Zhang, P. F. RSC Adv. 2016, 6, 37173.

[8] (a) Bellina, F.; Rossi, R. Chem. Rev. 2010, 110, 1082.

(b) Song, G.; Wang, F.; Li, X. Chem. Soc. Rev. 2012, 41, 3651.

(c) Gao, K.; Yoshikai, N. Acc. Chem. Res. 2014, 47, 1208.

(d) Yang, L.; Huang, H. M. Chem. Rev. 2015, 115, 3468.

(e) Yeung, C. S.; Dong, V. M. Chem. Rev. 2011, 111, 1215.

(f) Ackermann, L. Chem. Rev. 2011, 111,1315.

(g) Shen, C.; Zhang, P. F.; Sun, Q.; Bai, S. Q.; Hor, T. S. A.; Liu, X. G. Chem. Soc. Rev. 2015, 44, 291.

(h) Wang, X.-Y.; Gao, J.-Q.; Xu, X.-T.; Fang, P.; Mei, T.-S. Chin. J. Org. Chem. 2021, 41, 384 (in Chinese).

(王向阳, 高君青, 徐学涛, 方萍, 梅天胜, 有机化学, 2021, 41, 384.)

[9] For selected examples containing naphthalene derivatives as substrates in $\mathrm{C}-\mathrm{H}$ activation of arenes, see: (a) Stuart, D. R.; Bertrand-Laperle, M.; Burgess, K. M. N.; Fagnou, K. J. Am. Chem. Soc. 2008, 130, 16474.

(b) Wang, C.; Chen, H.; Wang, Z.; Chen, J.; Huang, Y. Angew. Chem., Int. Ed. 2012, 51, 7242.

(c) Li, Q.; Zhang, S.-Y.; He, G.; Ai, Z.; Nack, W. A.; Chen, G. Org. Lett. 2014, 16, 1764.

(d) Shi, S.; Kuang, C. J. Org. Chem. 2014, 79, 6105.

(e) Lee, D.; Chang, S. Chem.-Eur. J. 2015, 21, 5364.

(f) Kondrashov, M.; Raman, S.; Wendt, O. F. Chem. Commun. 2015, 51,911 .

(g) Moghaddam, F. M.; Tavakoli, G.; Saeednia, B.; Langer, P.; Jafari, B. J. Org. Chem. 2016, 81, 3868.

(h) Daugulis, O.; Zaitsev, V. G. Angew. Chem., Int. Ed. 2005, 44, 4046.

[10] (a) Huang, L.; Li, Q.; Wang, C.; Qi, C. J. Org. Chem. 2013, 78, 3030 .

(b) Odani, R.; Hirano, K.; Satoh, T.; Miura, M. J. Org. Chem. 2013, 78,11045 .

(c) Huang, L.; Sun, X.; Li, Q.; Qi, C. J. Org. Chem. 2014, 79, 6720. (d) Shang, R.; Ilies, L.; Nakamura, E. J. Am. Chem. Soc. 2015, 137, 7660 .

(e) Zhang, X.; Si, W.; Bao, M.; Asao, N.; Yamamoto, Y.; Jin, T. Org. Lett. 2014, 16, 4830 .

(f) Naksomboon, K.; Valderas, C.; Gómez-Martínez, M.; Álvarez-Casao, Y.; Fernández-Ibáñez, M. Á. ACS Catal. 2017, 7, 6342.
[11] (a) Ju, L.; Yao, J.; Wu, Z.; Liu, Z.; Zhang, Y. J. Org. Chem. 2013, 78,10821 .

(b) Roane, J.; Daugulis, O. Org. Lett. 2013, 15, 5842.

[12] (a) Pradhan, S.; De, P. B.; Punniyamurthy, T. J. Org. Chem. 2017, $82,4883$.

(b) Li, Z.; Su, S.; Qiao, H.; Yang, F.; Zhu, Y.; Kang, J.; Wu, Y.; Wu, Y. Org. Lett. 2016, 18, 4594

(c) Martínez, Á. M.; Rodríguez, N.; Arrayás, R. G.; Carretero, J. C. Chem. Commun. 2014, 50, 2801.

[13] (a) Xiong, Y. S.; Zhang, B.; Yu, Y.; Weng, J.; Lu, G. J. Org. Chem. 2019, 84, 13465 .

(b) Li, J. M.; Wang, Y. H.; Yu, Y.; Wu, R. B.; Weng, J.; Lu, G. ACS Catal. 2017, 7, 2661

[14] (a) Iwasaki, M.; Iyanaga, M.; Tsuchiya, Y.; Nishimura, Y.; Li, W.; Li, Z.; Nishihara, Y. Chem.-Eur. J. 2014, 20, 2459.

(b) Iwasaki, M.; Kaneshika, W.; Tsuchiya, Y.; Nakajima, K.; Nishihara, Y. J. Org. Chem. 2014, 79, 11330.

[15] Liang, S.; Bolte, M.; Manolikakes, G. Chem.-Eur. J. 2017, 23, 96;

[16] Bai, P.; Sun, S.; Li, Z.; Qiao, H.; Su, X.; Yang, F.; Wu, Y.; Wu, Y. J. Org. Chem. 2017,82, 12119.

[17] (a) Rosen, H.; Hajdu, R.; Silver, L.; Kropp, H.; Dorso, K.; Kohler, J.; Sundelof, J. G.; Huber, J.; Hammond, G. G.; Jackson, J. J.; Gill, C. J.; Thompson, R.; Pelak, B. A.; Epstein-Toney, J. H.; Lankas, G.; Wilkening, R. R.; Wildonger, K. J.; Blizzard, T. A.; DiNinno, F. P.; Ratcliffe, R. W.; Heck, J. V.; Kozarich, J. W.; Hammond, M. L. Science 1999, 283, 703.

(b) Kamal, A.; Ramakrishna, G.; Nayak, V. L.; Raju, P.; Rao, A. V. S.; Viswanath, A.; Vishnuvardhan, M. V. P. S.; Ramakrishna, S.; Srinivas, G. Bioorg. Med. Chem. 2012, 20, 789.

(c) Ilardi, E. A.; Vitaku, E.; Njardarson, J. T. J. Med. Chem. 2013, $57,2832$.

[18] (a) Chen, J.; Han, X.; Mei, L.; Liu, J.; Du, K.; Cao, T.; Li, Q. RSC Adv. 2019, 9, 31212 .

(b) Katrun, P.; Mueangkaew, C.; Pohmakotr, M.; Reutrakul, V.; Jaipetch, T.; Soorukram, D.; Kuhakarn, C. J. Org. Chem. 2014, 79, 1778.

[19] (a) da Silva Corrêa, C. M. M.; Waters, W. A. J. Chem. Soc. C 1968 1874.

(b) Truce, W. E.; Wolf, G. C. J. Org. Chem. 1971, 36, 1727.

(c) Truce, W. E.; Heuring, D. L.; Wolf, G. C. J. Org. Chem. 1974, 39,238

(d) Liu, L. K.; Chi, Y.; Jen, K.-Y. J. Org. Chem. 1980, 45, 406

(e) Harwood, L. M.; Julia, M.; Thuillier, G. L. Tetrahedron 1980 36, 2483.

(f) Barluenga, J.; Martínez-Gallo, J. M.; Nájera, C.; Yus, M.; Fananas, F. J. J. Chem. Soc., Perkin Trans. 1 1987, 2605.

(g) Nájera, C.; Baldó, B.; Yus, M.J. Chem. Soc., Perkin Trans. 1 $1988,1029$.

(h) daSilva Corrêa, C. M. M.; Fleming, M. D. C. M.; Oliveira, M. A. B. C. S. J. Chem. Soc., Perkin Trans. 2 1994, 1993.

(i) Nájera, C.; Sansano, J. M.; Yus, M. J. Chem. Educ. 1995, 72, 664.

(j) Craig, D. C.; Edwards, G. L.; Muldoon, C. A. Synlett 1997, 1441. 